\title{
DESign OF A CONSENSUS BASED FLOCKING CONTROL OF MULTIPLE AUTONOMOUS UNDERWATER VEHICLES USING SLIDING MODE APPROACH
}

\author{
Bidyadhar Subudhi ${ }^{1}$, Madan Mohan Rayguru ${ }^{1}$, \\ Vladimir Filaretov ${ }^{2,3}$, Aleksandr Zuev ${ }^{2,3}$ \\ ${ }^{1}$ Dept. of Electrical Engineering, National Institute of Technology, Rourkela-769008, India \\ ${ }^{2}$ Far Esatern Federal University, Vladivostok, Russia \\ ${ }^{3}$ Institute of Automation and Control Processes FEB RAS, Vladivostok, Russia
}
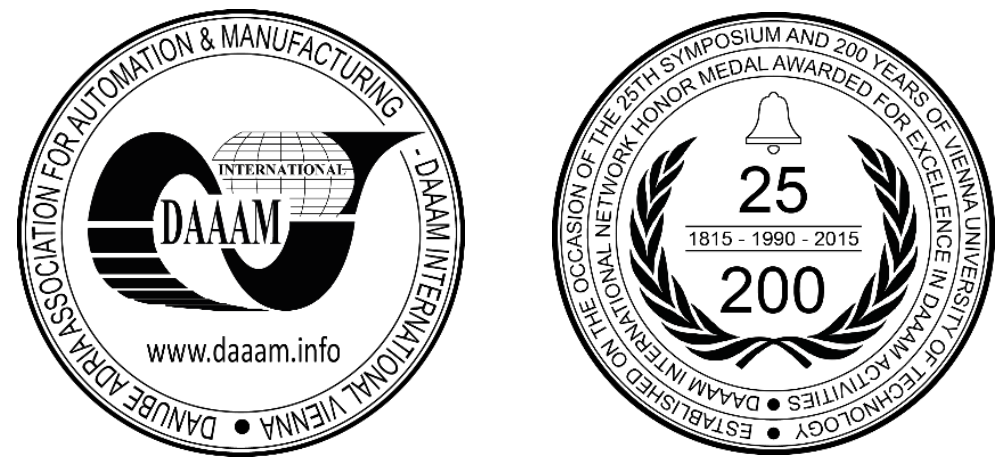

This Publication has to be referred as: Subudhi, B[idyadhar]; Rayguru, M[adan] M[ohan]; Filaretov, V[ladimir] \& Zuev, A[lexander] (2017). Design of a Consensus Based Flocking Control of Multiple Autonomous Underwater Vehicles Using Sliding Mode Approach, Proceedings of the 28th DAAAM International Symposium, pp.0004-0013, B. Katalinic (Ed.), Published by DAAAM International, ISBN 978-3-902734-11-2, ISSN 1726-9679, Vienna, Austria

DOI: $10.2507 / 28$ th.daaam.proceedings.001

\begin{abstract}
This paper proposes a flocking control algorithm for a team of autonomous underwater vehicles (AUVs) deployed for group motion in a network where only local communication exists among the participating AUVs. The control scheme is proposed with two levels where the inner level utilizes agreement or consensus protocol to derive reference positions for the follower AUVs whereas the outer loop employs a sliding mode controller (SMC) to reduce the tracking error to zero. SMC being a suitable candidate for handling is chosen because of its inherent robustness to any uncertainties and disturbances, it is intended to design a SMC to address the problem of handling uncertainties in the hydrodynamic parameters of the AUV dynamics and has been used to steer each AUV in the flock to their desired states. By exploiting the consensus protocol and SMC, it can be ensured that once an AUV comes into the neighbour zone (maximum range for information exchange for a member AUV outside which no communication is possible) cannot be separated from the group. Therefore, this controller structure is suitable for flocking of multiple AUVs where communication constraints play a vital role in efficient performance of control algorithms. The effectiveness of the proposed controller scheme is demonstrated through MATLAB simulation.
\end{abstract}

Keywords: AUV; Co-operative control; Flocking; Consensus; SMC

\section{Introduction}

Attention on cooperative control of robotic systems has grown enormously in the control community because of its increasing use in intelligence, surveillance, mine countermeasures, antisubmarine warfare, oceanography, payload 
delivery, information operations and time critical strikes [1-3]. Formation control is an approach to cooperative control strategy where a spatial structure of the group of agents/robots/AUVs in motion remains constant throughout the mission whereas flocking control is an approach where structure of the group in motion is dynamic or time varying. Flocking of multi agent systems with communication constraints is an emerging area of research now-a-days.

Formation behavior has been studied in robotics usually with different type structures namely behavior-based structure, leader-follower structure etc. [8, 17]. This work is concerned with leader follower structure only. Leader follower flocking is very similar to leader follower formation technique except that in the former the followers do not have to follow the leader with rigid position constraints [5]. However, the leader follower formation keeping leads to a poor disturbance rejection property due to absence of feedback from followers to leader [18]. By adding the algebraic graph theory and consensus protocol to the conservative control techniques we can confirm connectedness of flocking network and can model different communication topology with time varying structure easily [6, 7, 9-12]. Single and double integrator consensus has been effectively exploited in flocking of mobile robots and unmanned aerial vehicles (UAV) [12]. The flocking algorithms used in [8, 9, 12] composed of a feedback structure utilizing consensus protocol and derive control strategies using this feedback information to keep the participating members of the flock cohesive. These controller structures [24] are not effective in case of flocking of AUVs as these algorithms are not robust enough to uncertainties and nonlinearities present in the dynamics of the AUVs. One needs robust low-level controllers with high level planners using above cited algorithms so that the total structure become robust.

In recent years, leader following flocking control of multiple agents and UAVs has been developed using graph theory based control techniques [12]. Virtual leader based formation control methodology has been used to derive formation control for multiple AUVs [21]. In this approach approximation techniques are used to derive final control law which is not robust enough to parameter uncertainties. In potential energy based formation control techniques [22] also the controller is not robust to uncertainties. Some adaptive controllers [23] for formation keeping can be found in literature but these controllers are not derived using local information exchange between neighbor AUVs. However, a limited amount of literature is available on flocking of AUVs with limited communication facility which makes the information flow more complex and the required flocking controller should be able to sort out this problem while preserving connectedness. Therefore, flocking control of AUVs is a challenging task due to its non-linear dynamics and underwater communication constraint. A group of AUVs has been considered in which the leader only has the knowledge of the desired path. The problem is to make the group cohesive as the followers have the potential to diverge away from the group and to make the group follow a desired path.

The motivation of this paper lies in developing co-operative control algorithm of a group of AUVs where there is severe communication constraint compared to surface vessels. It is difficult to control a group of AUVs with low communication bandwidth because centralizing co-operative control techniques require information about the whole group at each instant and so it require a larger bandwidth which is not readily available in underwater environment. Therefore, the need of distributed control approach arises [4].

In this work a new flocking algorithm has been developed for a group of AUVs in network where only neighbor to neighbor (local) communication is available and the desired path is only known to the group leader. The new proposed flocking controller structure consists of two loops namely an inner loop one that calculates the reference position for the followers using single and double integrator consensus respectively. In the outer loop, sliding mode control is used to control each AUV to its desired track. Sliding mode control has been chosen for its robustness to any nonlinearity and uncertainties present in the AUV dynamics [13-16]. SMC is employed for tracking a reference path and distributed consensus is applied for determining the reference position for followers which is used for design of error vector and consequently sliding surface.

\section{Problem Statement}

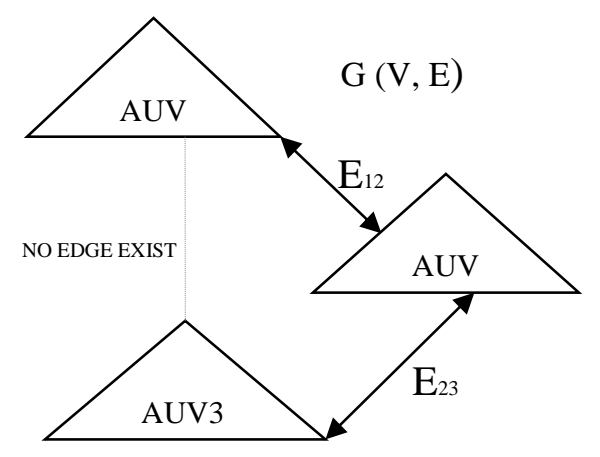

Fig. 1. Group of AUVs in a dynamic graph with each AUV represented as a node

Let $\mathrm{G}(\mathrm{V}, \mathrm{E})$ denotes a dynamic graph undirected graph represents a group of AUVs (consisting three AUVs) in a flock (see Fig. 1). Each AUV is represented as a node $V$ of this graph $G$ and an edge $E_{i j}$ exists between the $i^{\text {th }}$ node and $j^{\text {th }}$ 
node at any time instant if a network connection exists between the two AUVs present at those nodes. One of the AUVs in the group knows the desired path is termed as leader and other AUVs are termed as followers. The follower AUVs do not know which AUV of them is the leader and they do not know the desired path also. Let each AUV in the graph has a common dynamics of motion [1]. The objective is to develop a decentralized flocking control algorithm for the above group of AUVs where each AUV is controlled separately through a controller such that each of these AUVs remain in the flock and move in a predefined path with common forward velocity. In flocking problem for a group of AUVs, one important task is to achive convergence of the heading angle of each AUV in the group so that they will follow the same path keeping forward velocity constant.

\section{System Description}

The motion of the body-fixed frame of reference is described relative to an inertial or earth-fixed reference frame (Fig. 2).

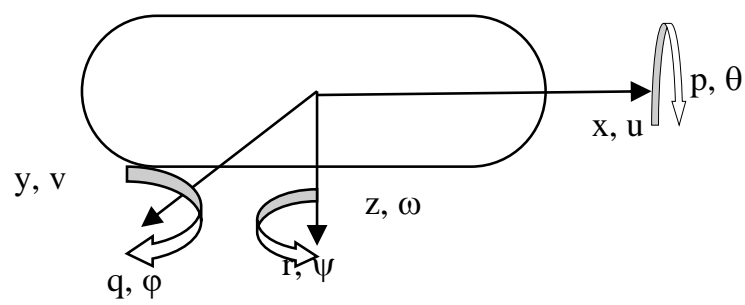

Fig. 2. Degrees of freedom of AUV

The notations different variables used are defined as follows:

$\mathrm{x}, \mathrm{u} \quad$ are inertial $\mathrm{x}$ coordinate position and body fixed surge velocity;

$\mathrm{y}, \mathrm{v} \quad$ are inertial y coordinate position and body fixed sway velocity;

$\mathrm{z}, \omega \quad$ are inertial $\mathrm{z}$ coordinate position and body fixed heave velocity;

$\mathrm{p}, \theta \quad$ are angular orientation (pitch) and rate of change of orientation along $\mathrm{x}$ axis;

$\mathrm{q}, \varphi \quad$ are angular orientation (roll) and rate of change of orientation along y axis;

$\mathrm{r}, \psi \quad$ are angular orientation (yaw) and rate of change of orientation along $\mathrm{z}$ axis.

The position and orientation are described in earth fixed reference frame and velocities (both linear angular) are described in body fixed reference frame. The equations of motion of an AUV in x-y plane can be written as (neglecting pitch and roll):

$\underbrace{\left[\begin{array}{cccccc}m-X_{\dot{u}} & 0 & 0 & 0 & 0 & 0 \\ 0 & m-Y_{\dot{v}} & 0 & 0 & 0 & 0 \\ 0 & 0 & I_{z z}-N \dot{r} & 0 & 0 & 0 \\ 0 & 0 & 0 & 1 & 0 & 0 \\ 0 & 0 & 0 & 0 & 1 & 0 \\ 0 & 0 & 0 & 0 & 0 & 1\end{array}\right]}_{M} \underbrace{\left[\begin{array}{c}\dot{u} \\ \dot{v} \\ \dot{r} \\ \dot{\psi} \\ \dot{x} \\ \dot{y}\end{array}\right]}_{\dot{X}}=$

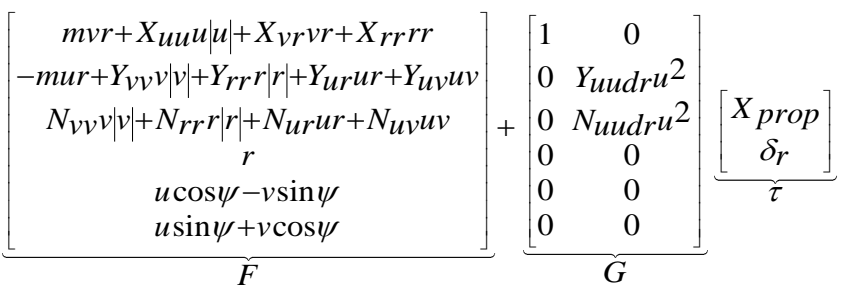

$Y=\underbrace{\left[\begin{array}{llllll}0 & 0 & 0 & 0 & 1 & 0 \\ 0 & 0 & 0 & 0 & 0 & 1\end{array}\right]}_{H}[x], X=[u, v, r, \psi, x, y]^{T}$

where $Y$ is the otput matrix, $X_{\text {prop }}$ is the thruster force, $\delta$ is the rudder angle, $m$ is the mass of the vehicle, $I_{z z}$ is moment of inertia along $\mathrm{z}$ axis and other parameters are known as hydrodynamic parameters are given in appendix. AUVs in flock are free to move without rigid position constraints so they can move sidewise (x-y plane) and in flock they always maintain a minimum distance among them. Therefore even if the flocking algorithm does not consider z-plane motion, the flock remains intact. 


\section{Control Law Development}

The controller structure is presented in Fig. 3; it is divided in two levels. The group of AUVs which are in the flock can communicate locally so they can transmit their position and velocity $\left(x_{i}, y_{i}, u_{i}, v_{i}\right)$ to the local neighbors. Each AUV can determine the desired position and velocity to remain in the flock using this transmitted information from neighbors using consensus algorithm. Finally using the desired states a feedback control law for each AUV is developed through sliding control law.

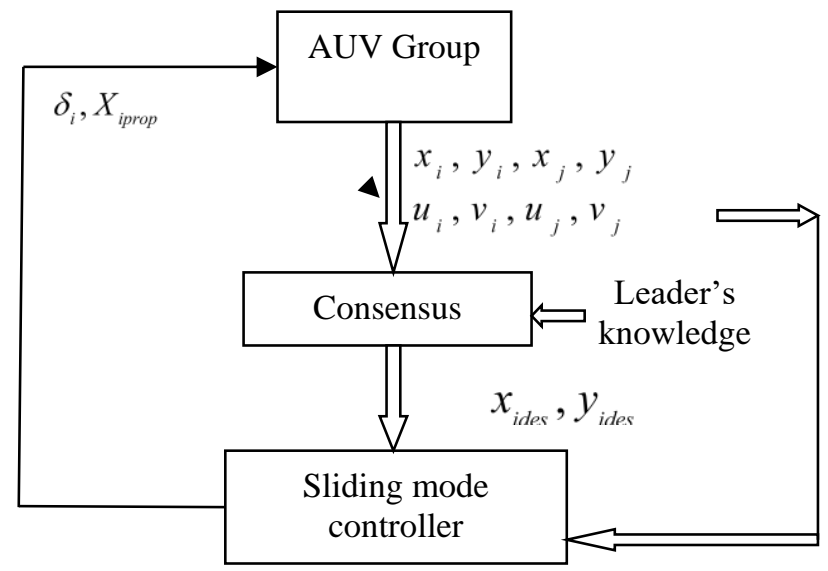

Fig. 3. Controller structure for flocking of AUVs

\subsection{Sliding Surface Design}

Dynamics of AUV in (1) can be written as

$$
M \dot{X}=F(X)+G(X, \tau)
$$

The sliding surface is chosen as

$$
\sigma=\tilde{Y}+\gamma \dot{\tilde{Y}}, \text { where } \gamma=\left[\begin{array}{cc}
\gamma_{1} & 0 \\
0 & \gamma_{2}
\end{array}\right]
$$

For leader $\operatorname{AUV}\left(\tilde{Y}=H X-Y_{d}\right)$ and follower AUVs $\left(\tilde{Y}=H X-Y_{d f}\right) \mathrm{Y}_{\mathrm{d}}$ is the desired track chosen. The leader leader AUV should know the desired track but the desired track $\mathrm{Y}_{\mathrm{df}}$ for followers is derived from consensus algorithm. $\gamma$ is chosen as diagonal matrix whose elements are positive constants and it weights the velocity error term compared to position error. It has a direct effect on convergence of state dynamics into sliding surface.

\subsection{Graph Theory and Laplacian}

A network of group of agents can be modeled using algebraic graph theory. Graph can be used to represent interconnections between nodes. A vertex of the graph corresponds to a node, and the edges of the graph capture the dependence of interconnections.

Formally, a graph $G=(V, E)$ consists of a set of vertices, $V=[1,2 \ldots N]$ and set of edges $\mathrm{E}=\{(\mathrm{i}, \mathrm{j}) \in \mathrm{V} \times \mathrm{V}\}$ containing unordered pairs of distinct vertices. The graph has no self-loop, i.e., (i, j) $\in E$ implies $i \neq j$. A graph is connected if, for any vertices $(\mathrm{i}, \mathrm{j}) \in \mathrm{V}$, there exists a path of edges in $\mathrm{E}$ from to $\mathrm{i}$ to $\mathrm{j}$. The set of neighbors of vertex i is defined as $N_{i}=$ $\{\mathrm{j} \in \mathrm{V}:(\mathrm{i}, \mathrm{j}) \in \mathrm{E}\}$.

The degree of vertex is defined as $d_{i}=N_{i}$, and the maximum degree is $d_{\max }=\max \left(d_{i}\right)$. Let D be the degree matrix [ $\left.\mathrm{D}=\operatorname{diag}\left(d_{i}\right)\right]$. The adjacency matrix $\mathrm{A}$ is the integer matrix with rows and columns indexed by the vertices, e.g., $\mathrm{i}-\mathrm{j}$ entry of $\mathrm{A}$ is equal to the number of edges from $\mathrm{i}$ to $\mathrm{j}$. The Laplacian matrix $\mathrm{L}$ of a graph $\mathrm{G}$ is defined as $\mathrm{L}=\mathrm{D}-\mathrm{A}$

For a connected graph, the Laplacian matrix $\mathrm{L}$ is symmetric and positive semi definite. Its minimum eigenvalue is zero, and the corresponding eigenvector is $[1,1 \ldots 1]^{\mathrm{T}}$. 


\subsection{Command Angle and Reference Speed Generation}

Consensus or agreement protocol can easily model a network of multiple agents without really going into the detail about information flow between the agents. The network is modeled as a undirected graph (weighted) or as a directed graph. Participating agents in the flock can be viewed as nodes where as their information flow with their neighbors are represented with directed or undirected edges having specific weights. Control strategy applied for flocking control uses the information states in consensus to design feedback structure for formation tracking, maneuvering etc. This algorithm adapts the first derivative of the agreement information state on each node based on the value of the agreement variable of its neighbors. In this work we use the consensus algorithm to derive the desired heading for followers and apply a sliding mode control strategy to generate control input (in this case rudder angle). This idea is helpful when communication bandwidth is very small and only local neighbor to neighbor communication is possible (Information constrained network).

Desired position and rate of its change for the followers are decided by single integrator consensus and double integrator consensus algorithms. Consensus algorithm abstracts the communication topology in to a simple information sharing protocol in a static or dynamic network. The convergence of information exchange occurs if there is a minimal spanning tree always present in the dynamic graph [17]. As the problem defined in this paper is to converge the position of each AUV in the group to the desired position which is given only to the leader. If the dynamics of AUV is neglected then the output of each AUV should change according to eq. (5), (6) to reach consensus among the group [14, 19].

$$
\begin{aligned}
& \left.\dot{Y}_{i d}=-\sum_{j \in N}\left(Y_{i d}-Y_{j d}\right)-\left(\alpha_{i}-\alpha_{j}\right)\right) \\
& \ddot{Y}_{i d}=-\sum_{j \in N}\left(Y_{i d}-Y_{j d}\right)-\sum_{j \in N} \beta_{i j}\left(\dot{Y}_{i d}-\dot{Y}_{j d}\right)
\end{aligned}
$$

The equation described above are extensions of single and double integrator consensus algorithms which are thoroughly discussed in various literature [19-21]. These equations give a desired rate of change of output position for the follower AUVs and a sliding controller will be used to force the actual position of each AUV to their desired one. Let's $Y_{i d}$ is the desired position for follower $i$ and is chosen from eq. (5) and (6), $N$ is the neighbor set of $i^{\text {th }}$ AUV in a dynamic graph consisting of all participating AUVs. $\alpha_{\mathrm{i}}-\alpha_{\mathrm{j}}$ is the required difference in position between $\mathrm{i}^{\text {th }}$ and $\mathrm{j}^{\text {th }}$ AUV for avoiding collision and $\beta_{\mathrm{ij}}$ is a gain which is arbitrary chosen as 1 here [19], [20]. By the consensus algorithm given by eq. (5) difference between information state of $i_{\text {th }}$ and $j_{\text {th }}$ AUV will converge to zero [19].

$$
\sum_{t \rightarrow \infty} Y_{i d}-Y_{j d}=\alpha_{i}-\alpha, \sum_{t \rightarrow \infty} \dot{Y}_{i d}-\dot{Y}_{j d}=0
$$

Theorem 1: The consensus eq. (5) will converge asymptotically if and only if a minimal spanning tree always exist in the information exchange topology [19]

Proof: Eq. (5) can be written in the form of [19]

$$
\begin{aligned}
& \dot{Y}_{i d}=-\sum_{\mathrm{j} \in \mathrm{N}}\left(\mathrm{Y}_{i d}^{*}-\mathrm{Y}_{j d}^{*}\right), \mathrm{Y}_{i d}^{*}=\mathrm{Y}_{\mathrm{id}}-\alpha_{\mathrm{i}}, \mathrm{Y}_{j d}^{*}=\mathrm{Y}_{\mathrm{jd}}-\alpha_{\mathrm{j}} \\
& \dot{Y}_{d}=-L Y_{d} \\
& \mathrm{Y}_{d}=\left[\mathrm{Y}_{i d}^{*}, \mathrm{Y}_{j d}^{*}, \ldots . .\right]^{\mathrm{T}}
\end{aligned}
$$

We have taken the network graph of AUVs to be connected, for this case the solution to eq. (8) is

$$
Y(t)=e^{L t} Y(0)
$$

Time is denoted as ' $t$ ' and The spectrum of Laplacian matrix ' $L$ ' of a undirected connected sub graph is [20]

$$
0=\lambda_{1}<\lambda_{2} \leq \ldots \ldots \leq \lambda_{N}
$$

The lowest eigen value $\lambda_{1}$ which is zero corresponds to a eigen vector 1 , the vector of all ones. $\mathrm{L}$ is symmetric and $\mathrm{L} 1=0$ for an arbitrary undirected $G$. Let the matrix $\mathrm{L}$ has mutually orthogonal and normalized eigen vectors $\mathrm{Q}=\left[\mathrm{Q}_{1} \mathrm{Q}_{2}\right.$ .. $\left.\mathrm{Q}_{\mathrm{N}}\right]$ corresponding to its ordered eigen values. Furthermore, set

$$
\Lambda=\operatorname{Diag}\left(\lambda_{1}, \lambda_{2}, \ldots \ldots \lambda_{N}\right)
$$




$$
\mathrm{Y}(\mathrm{t})=e^{-\lambda_{1} t}\left(Q_{1}^{T} Y_{0}\right) Q_{1}+e^{-\lambda_{2} t}\left(Q_{2}^{T} Y_{0}\right) Q_{2}+\cdots e^{-\lambda_{N} t}\left(Q_{N}^{T} Y_{0}\right) Q_{N}
$$

For a connected graph $\lambda_{1}$ is zero and $\lambda_{i} \geq 0$ for $i \geq 2$ therefore as [20]

$$
t \rightarrow \infty, Y(t) \rightarrow Q_{1}^{T} Y_{0} Q_{1}=\frac{1^{T} Y_{0}}{N} 1
$$

and $\lambda_{2}$ the smallest positive value of graph Laplacian dictates the slowest mode of convergence. As a positive value of $\lambda_{2}$ suffice the asymptotic convergence and connectivity it can be concluded that a minimum order structure needed for convergence of the equation is a graph containing a spanning tree. Similarly eq. (6) can be written as

$$
\ddot{Y}=-L Y-\beta L \dot{Y}
$$

$\mathrm{L}=\mathrm{Laplacian}$ matrix of dynamic graph (flocking network) and $\beta=\operatorname{diag}\left(\boldsymbol{\beta}_{i j}\right)$.

Convergence of consensus algorithm requires [12] a spanning tree to be present in the information topology and the weighting factors $(\beta)$ should be greater than some constant which is given by

$$
\begin{aligned}
& \beta_{i j}>\max _{i=2, N N} \sqrt{\frac{2}{\left|\lambda_{i}\right| \cos \left(\frac{\pi}{2}-\tan ^{-1} \frac{\operatorname{Re}\left(\lambda_{i}\right)}{\operatorname{Im}\left(\lambda_{i}\right)}\right)}} \\
& Y_{i}-Y_{j} \rightarrow 0, \dot{Y}_{i}-\dot{Y}_{j} \rightarrow 0, \forall i \neq j, \text { as } t \rightarrow \infty \text { for any } Y_{i}(\mathrm{O}), \dot{Y}_{i}(\mathrm{O})
\end{aligned}
$$

\subsection{Control Law Development}

A sliding control law should be chosen such that the rate of change of state vector of the AUV system in the flock will always point to the sliding surface. So that once in the vicinity of the sliding surface it will always remain inside this surface. A Lyaponov stability criterion is used to generate a control law to stabilize the plant (AUV) in case of uncertainties. Let $\mathrm{V}$ is a Lyaponov candidate function defined as

$$
\mathrm{V}=\frac{1}{2} \sigma^{2}
$$

Then asymptotic stability of error vector between actual states and reference states of the AUV is guaranteed if [14]

$$
\dot{V} \leq-\mu^{2} \operatorname{sign}(\sigma)
$$

where $\mu$ is a positive quantity which should be chosen properly by trade of between magnitude of control forces generated and rate of convergence of state dynamics of each AUV into the sliding plane. A higher value would generate a higher magnitude of control action which is always undesired. Using eq. (4) the dynamics of leader AUV in the sliding plane is [14]

$$
\left.\dot{\sigma}=\dot{\tilde{Y}}-\ddot{\widetilde{Y}}=H \dot{X}-\dot{Y}_{d}\right)+\gamma\left(H \ddot{X}-\ddot{Y}_{d}\right)
$$

For follower AUVs the sliding plane is derived from eq. (5) and (6)

$$
\dot{\sigma}=\dot{\tilde{Y}}-\ddot{\widetilde{Y}}=\left(H \dot{X}-\dot{Y}_{i d}\right)+\gamma\left(H \ddot{X}-\ddot{Y}_{i d}\right)
$$

To reduce chattering a boundary layer B with a continuous hyperbolic tangent function is used in place of hard limiting signum function

$$
\begin{aligned}
\dot{\sigma} & =\left(H\left(M^{-1} F(X)+M^{-1} G \tau\right)-\dot{Y}_{d}\right)+\gamma\left(H\left(M^{-1} \dot{F}(X)+M^{-1} G \dot{\tau}+M^{-1} \dot{G} \tau\right)-\ddot{Y}_{d}\right) \\
& =-\mu^{2} \tanh \left(\frac{\sigma}{\mathrm{B}}\right)
\end{aligned}
$$


$H M^{-1} G=H M^{-1} \dot{G}=\left[\begin{array}{ll}0 & 0 \\ 0 & 0\end{array}\right]^{-1}$

$\dot{F}(X)=\frac{\partial F}{\partial X} \frac{\partial X}{\partial t}$

$\dot{F}(X)=\frac{\partial F}{\partial X} \dot{X}$

$\frac{\partial F}{\partial X}=F_{d}=\left[\begin{array}{ll}F_{d 1} & F_{d 2} \\ F_{d 3} & F_{d 4}\end{array}\right]$

$F_{d 1}=\left[\begin{array}{ccc}2 X_{u u} u & m r+X_{v r} r & m v+X_{v r} v+2 X_{r r} r \\ -m r+Y_{u r} r+Y_{u v} v & 2 Y_{v v} v+Y_{u v} u & -m u+2 Y_{r r} r+Y_{u r} u \\ N_{u v} v & 2 N_{v v} v+N_{u v} u & 2 N_{v r} r+N_{u r} u\end{array}\right]$

$F_{d 2}=\operatorname{zeros}(3 \times 3)$

$F_{d 3}=\left[\begin{array}{ccc}0 & 0 & 1 \\ \cos \psi & -\sin \psi & 0 \\ \sin \psi & \cos \psi & 0\end{array}\right]$

$F_{d 4}=\left[\begin{array}{ccc}0 & 0 & 0 \\ 0 & -u \sin \psi-v \sin \psi & 0 \\ 0 & u \cos \psi-v \sin \psi & 0\end{array}\right]$

From eq. (20), (21) and (23)

$$
\tau=\left(\lambda H M^{-1} \dot{F}(X) M^{-1} G\right)^{-1}\left[\left(\dot{Y}_{d}-H M^{-1} F(X)\right)+\gamma\left(\ddot{Y}_{d}-H M^{-1} \dot{F}(X) M F\right)-\mu^{2} \tanh \left(\frac{\sigma}{B}\right)\right]
$$

The sliding mode controller developed above can be made robust enough to deal with uncertainties present in the model.

\section{Results and Discussion}

Effectiveness of the proposed SMC flocking control of multiple AUVs is presented in this section. A flocking situation is simulated considering three similar REMUS AUVs. The parameters used are same as in Prestro et al [1]. The kinematic and dynamic equations of the AUVs given in eq. (1) and (2) are used to apply the proposed control eq. (26).

The simulations were performed by using MATLAB and SIMULINK. In this paper we have chosen a local communication radius of a distance $3 \mathrm{~m}$. That means whenever two AUVs come within this range only local communication is possible and outside this range no connection exist. An edge exists between two nodes of the graph iff distance between them is less than 3 meter. Other factors are chosen as follows:

$$
\begin{aligned}
\lambda & =\left[\begin{array}{ll}
2 & 0 \\
0 & 2
\end{array}\right] & \phi & =\left[\begin{array}{ll}
5 & 0 \\
0 & 5
\end{array}\right] \\
\mu & =\left[\begin{array}{cc}
40 & 0 \\
0 & 40
\end{array}\right] & Y_{d} & =\left[\begin{array}{l}
20 \cos (0.1 t) \\
20 \sin (0.1 t)
\end{array}\right]
\end{aligned}
$$

where $\lambda$ is chosen as diagonal matrix and it weights the velocity error term compared to position error. This parameter decides the position or its rate dominant the sliding surface and it has a direct effect on time constant of particular output variable. The smoothening constant ' $\phi$ ' should be chosen as a tradeoff between robustness and high frequency switching which can lead to excitation of unmodeled dynamics. 


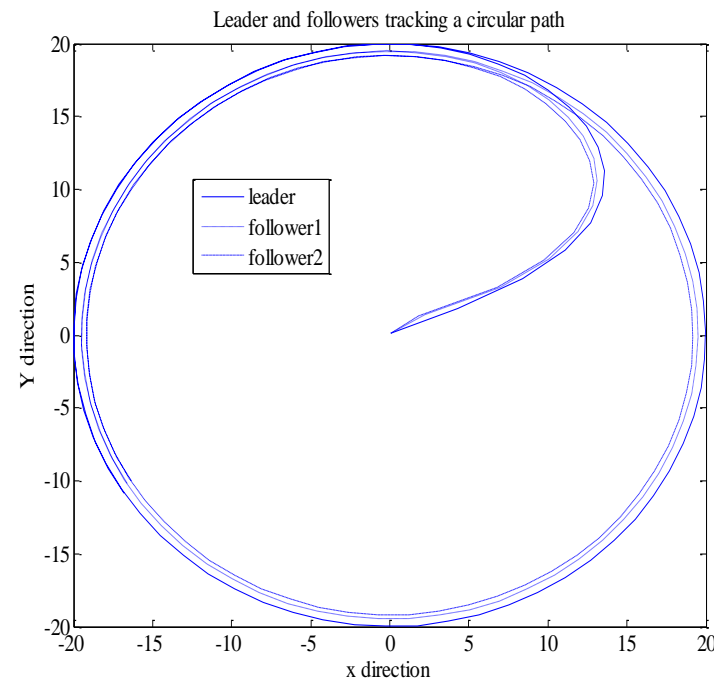

Fig. 4. Tracking of a circular path

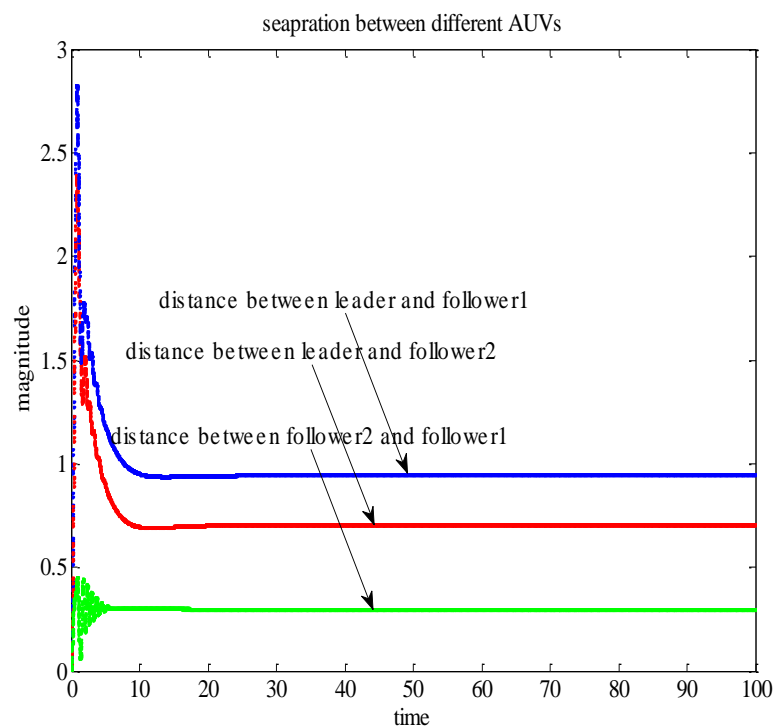

Fig. 5. Distance between the AUVs during flocking motion

A circular path of radius $20 \mathrm{~m}$ is specified to the leader. The proposed flocking controller is able to keep the group together with tracking the desired track. A separation of $1 \mathrm{~m}$ is used in consensus protocol which combined with sliding mode controller was successful in keeping the AUVs separate at desired distance.

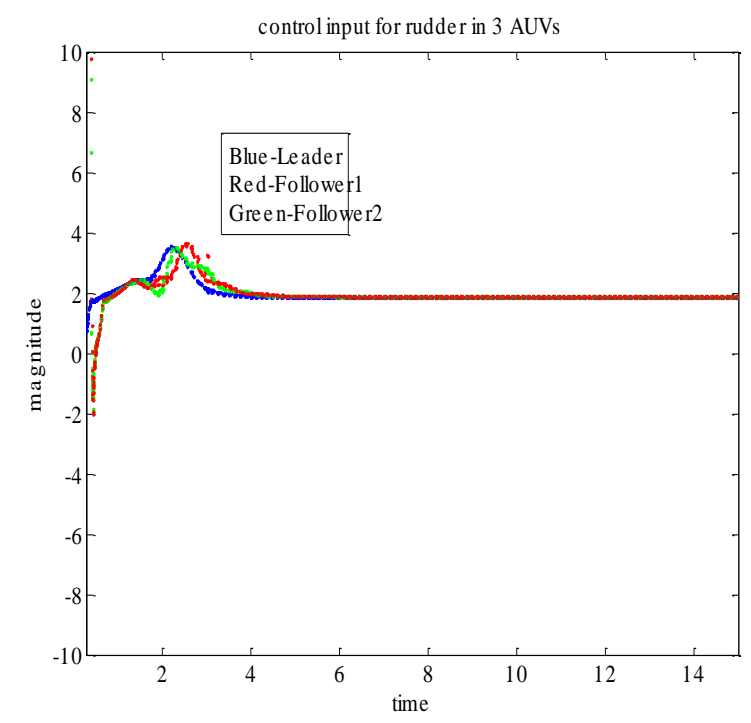

Fig. 6. Rudder angle input for three AUVs

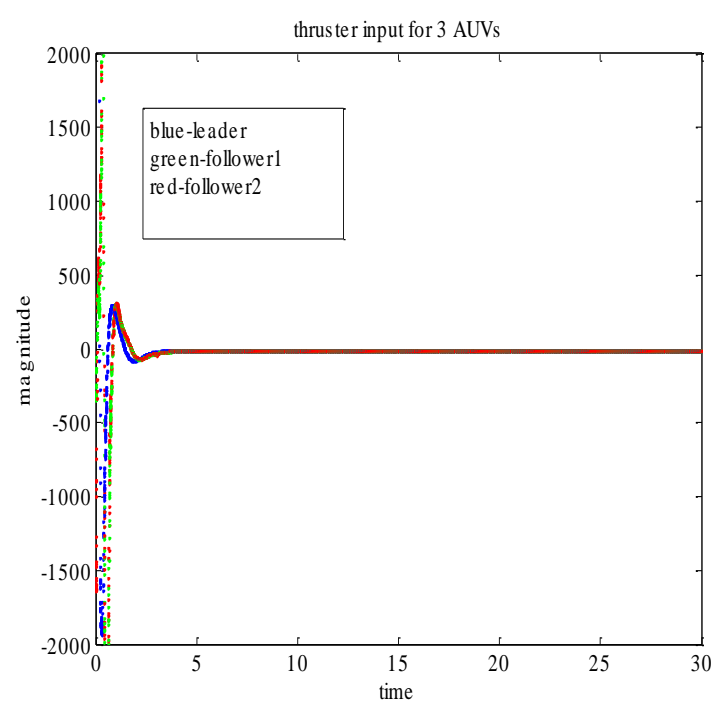

Fig. 7. Thruster input for three AUVs

Initially the thruster forces and rudder inputs required as shown in Fig. 6 and Fig. 7 are high but practically realizable and after the AUVs hit the sliding surface the control inputs required are pretty much small. In above simulation eq (7) and (26) are utilized to follow a circular track of radius $20 \mathrm{~m}$.

From the simulation result, it is verified that a constant rudder angle is generated from the proposed controller to follow a circular track and meanwhile the thruster input is of a constant magnitude so that the AUVs track the desired track in approximately constant speed.

Fig.8, Fig.9 and Fig. 10 envisage that the proposed controller provide constant angular velocity (0.1), constant surge and sway velocities for tracking a circular path even with uncertainty in the model. 


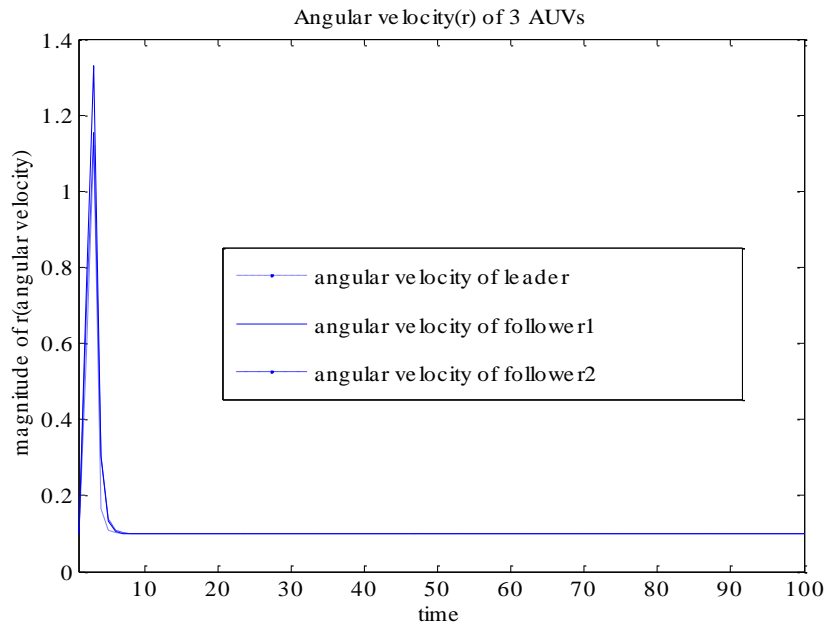

Fig. 8. Angular velocity while flocking for 3 AUVs

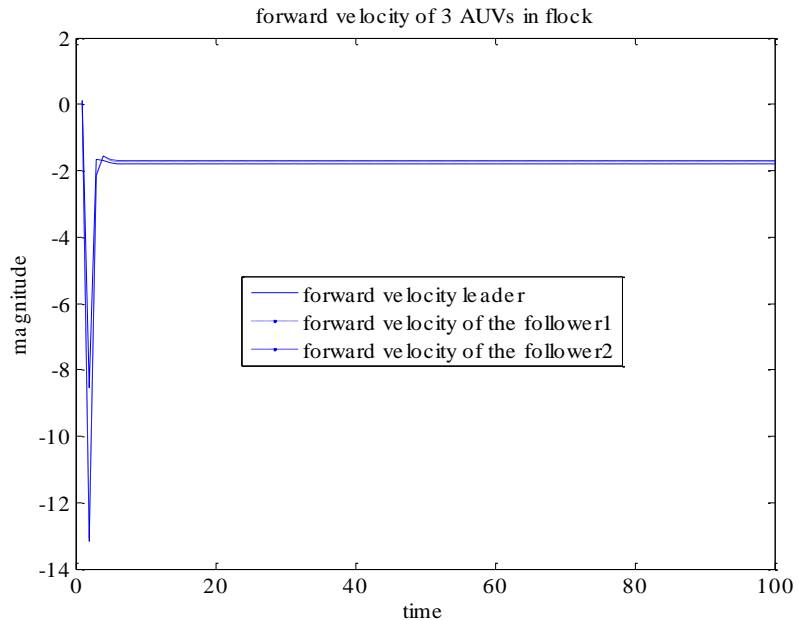

Fig. 9. Surge velocity while flocking for 3 AUVs

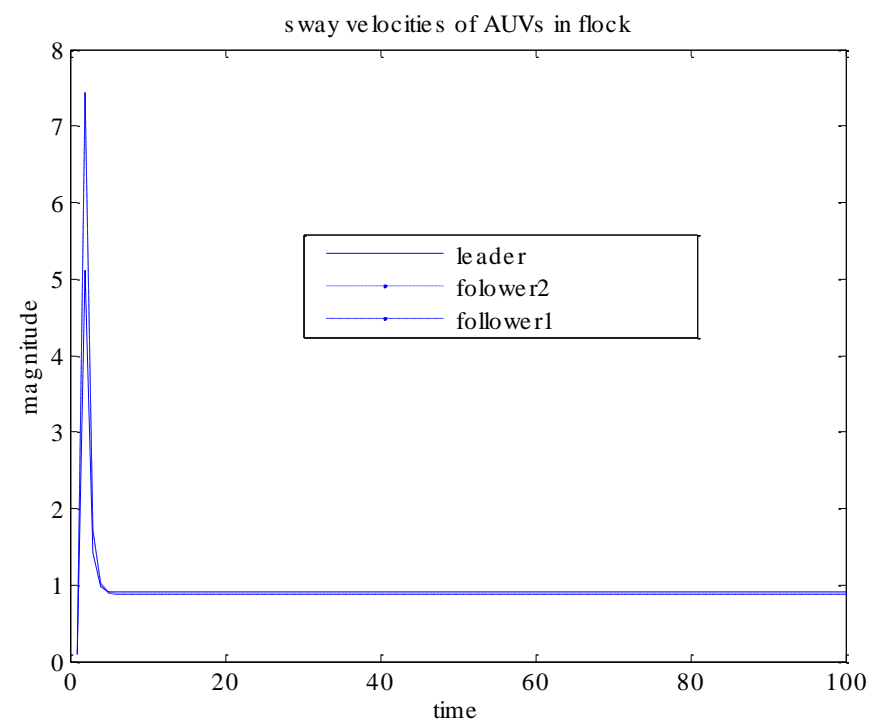

Fig. 10. Sway velocity while flocking for 3 AUVs

\section{Conclusions}

We proposed a sliding mode approach to design a flocking control algorithms for achieving an effcient cooperative motion of three AUVs. We have demnostaed that by employing consensus algorithm in the feedback structure provides a simple but effective tool for maintaining a flock of AUVs with local communication facilities. The proposed new SMC based robust controller provides excellent leader follower flocking performance. This control schem is not only simple but also robust enough to handle a large amount of uncertainties present in the AUV dynamics. Consensus algorithm (single and double integrator) is exploited for generation of the reference command for the follower AUVs in an information constrained network (local communication and low bandwidth) whereas sliding mode control handles robustness and nonlinearity present in the AUV dynamics.

Future work will focus in designing a switching network and deal with communication loss in the flocking control.

\section{Acknowledgments}

This research was supported by joint Russian-Indian project (RFBR 17-57-45055) and project MK-8536.2016.8.

\section{References}

[1] Timothy, P. (2001). Verification of a six-degree of freedom simulation model of REMUS autonomous underwater vehicle, M.S. Dissertation, Department of Ocean Engineering, Massachusetts Institute of Technology and Dept. of Mechanical Engineering, Woods Hole Oceanographic Institution. 
[2] Filaretov, V.; Konoplin, A., Konoplin, N. \& Gorbachev, G. (2016). Control system for underwater vehicle with multilink manipulator for automatic manipulation operations, Proceedings of the 27th DAAAM International Symposium, 2016, Vienna, Austria, ISBN 978-3-902734-08-2, ISSN 1726-9679, Katalinic, B. (Ed.), pp.0714-0720, DOI: $10.2507 / 27$ th.daaam.proceedings.103

[3] Filaretov, V. \& Pryanichnikov, V. (2015) Autonomous mobile university robots amur: technology and applications to extreme robotics. Procedia Engineering, Vol. 100, (2015), pp. 269-277, ISSN 1877-7058.

[4] Winfield, A. (2000). Distributed sensing and data collection via broken ad hoc wireless connected networks of mobile robots, In: Distributed Autonomous Robotic Systems 4, L.E. Parker, G. Bekey and J. Barhen, (Ed.), 273282, Springer-Verlag, ISBN 978-4-431-67919-6, New York.

[5] Desai, J. P., Kumar, V. \& Ostrowski, J. P. (2001). Modeling and control of formations of nonholonomic mobile robots. IEEE Transactions on Robotics and Automation, Vol. 17, No. 7, (2001), pp. 905-908, ISSN 1042-296X.

[6] Fax, J. A. \& Murray, R. M. (2004). Information flow and cooperative control of vehicle formations. IEEE Transactions on Automatic Control, Vol. 49, No. 9, (2004), pp.1465-1476, ISSN 0018-9286.

[7] Das, B., Subudhi, B. \& Pati, B. B. (2016). Adaptive sliding mode formation control of multiple underwater robots. Archives of Control Sciences, Vol. 24(LX), No. 4, (2014), pp. 515-543, ISSN 2300-2611.

[8] Gu, D. \& Wang Z. (2009). Leader-follower flocking: algorithms and experiments. IEEE Transactions on Control Systems Technology, Vol. 17, No. 5, (2009), pp. 1211 - 1219, ISSN 1063-6536.

[9] Ji, M. \& Egerstedt, M. (2007). Distributed coordination control of multi agent systems while preserving connectedness. IEEE Transactions on Robotics, Vol. 23, No. 4, (2007), pp. 693 - 703, ISSN 1552-3098.

[10] Tanner, H. G., Jadbabaie, A. \& Pappas, G. J. (2007). Flocking in fixed and switching networks. IEEE Transactions on Automatic Control, Vol. 52, No. 5, (2007), pp.863-868, ISSN 0018-9286.

[11] Das, B., Subudhi, B. \& Pati, B. B. (2016). Co-operative control coordination of a team of underwater vehicles with communication constraints. Transactions of the Institute of Measurement and Control, Vo. 38, No. 4, (2016), pp. 463-481, ISSN 1477-0369.

[12] Ren, W., \& Atkins, E.M. (2007). Distributed multi-vehicle coordinated control via local information exchange. International Journal of Robust and Nonlinear Control, Vol. 17, (2007), pp. 1002-1033, ISSN 1099-1239.

[13] Ren, W. (2007). Distributed attitude alignment in spacecraft formation flying. International journal of adaptive control and signal processing, Vol. 21, No. 2-3, (2007), pp.95-113, ISSN 1099-1115.

[14] Fahimi, F. (2007). Sliding-mode formation control for under actuated surface vessels. IEEE Transactions on Robotics, Vol.23, No.3, (2007), pp.617-622, ISSN 1552-3098.

[15] Healey, A J. \& Lienard, D. (1993). Multivariable sliding mode control for autonomous diving and steering of unmanned underwater vehicles. IEEE Journal of Oceanic Engineering, Vol. 18, No. 3, (1993), pp. 1-13, ISSN 03649059.

[16] Utkin,V. I. (1977). Variable structure systems with sliding modes. IEEE Transactions on Automatic Control, Vol. 22 No. 2, (1977), pp.212-222, ISSN 0018-9286.

[17] Balch, T. \& Arkin, C. (1998). Behaviour based formation control of multi robot teams. IEEE Trans. on Robotics and Automation, Vol.14, No. 2, (1998), pp. 926 - 939, ISSN 1042-296X.

[18] Das, B., Subudhi, B. \& Pati, B. B. (2016). Co-operative formation control of autonomous underwater vehicles: an overview. International Journal of Automation and computing, Vo. 13, No. 3, (2016), pp. 199-225, ISSN $1751-8520$.

[19] Ren, W. (2008). On consensus algorithms for double-integrator dynamics. IEEE Transactions On Automatic Control, Vol. 53, No. 6, (2008), pp. 1503 - 1509, ISSN 0018-9286.

[20] Zhu, J., Tian, Y. \& Kuang, J. (2009). On the general consensus protocol of multi-agent system with double integrator dynamics. Linear Algebra and its Applications, Vo. 431, No. 5-7, (2009), pp. 701-715, ISSN 0024-3795.

[21] Cui, R., Ge, S. S. \& How, B. V. E. (2010). Leader-follower formation control of under actuated autonomous underwater Vehicles. Ocean Engineering, Vol. 37, No. 17-18, (2010), pp. 1491-1502, ISSN 0029-8018.

[22] Das, B., Subudhi, B. \& Pati, B. B. (2016). Co-operative control of a team of autonomous underwater vehicles in an obstacle-rich environment. Journal of Marine Engineering \& Technology, Vol. 15, No. 3, (2016), pp. 135-151, ISSN 2046-4177.

[23] Das, B., Subudhi, B. \& Pati, B. B. (2016). Employing nonlinear observer for formation control of AUVs under communication constraints. International Journal of Intelligent Unmanned Systems, Vol. 3, No. 2/3, (2015), pp. 122-155, ISSN 2049-6427.

[24] Subudhi, B., Mukherjee, K. \& Ghosh, S. (2013). A static output feedback control design for path following of autonomous underwater vehicle in vertical plane. Ocean Engineering, Vol. 63, No. 1, (2013), pp. 72-76, ISSN 00298018 . 